\title{
Performance of downlink schedulers with superposed or orthogonal transmissions
}

\author{
Adrian Agustin, Member IEEE, Josep Vidal, Member IEEE and Olga Muñoz
}

\begin{abstract}
This work looks into the extension of the proportional fair (PF) scheduler to the multi-user case, where the source is transmitting several messages under the superposition coding (SC) strategy in a Gaussian Broadcast channel. Jointly with the weighted sum-rate (WSR) scheduling criterion, we derive guidelines for 2-user pairing, based both on SNR and user priority. We compare superposition coding access with frequency division multiple access, while time division is obtained naturally as a result of the user pairing. Results elucidate that the 2-user SC PF always improves the single-user PF in terms of average throughput and delay reduction, and we compare its performance with the 3-user SC PF. Finally, the WSR shows throughput gains similar to the 2-user SC PF for certain configuration of the scheduler.
\end{abstract}

Index Terms - Scheduling, proportional fair, multiuser, broadcast channel

\section{INTRODUCTION}

Wireless networks must support a large type of services, differing in throughput and delay constraints. Because of the scarcity of the wireless resources, its use must be efficiently managed. In [1] it was shown that the system throughput can be maximized by exploiting multi-user diversity, i.e. selecting the user with the best channel condition. However, such greedy scheduler is unfair as some users may never be served.

The criterion of proportional fairness (PF) is introduced in [2] in order to deal with the tradeoff between the maximum system throughput and user fairness. One example of singleuser PF is the scheduler of the Qualcomm's High Data Rate (HDR) system [3], where the user with the best metric based on channel state and average served throughput is selected. Enhancements of such system for static propagation scenarios can be found in [4], where multiple antennas are employed to induce large and fast channel fluctuations in slow-fading scenarios (opportunistic beamforming). Likewise, many others criteria for designing the throughput and delay of a network have been proposed, like max-min and the max sum-rate, [5], the weighted PF, [6], or the weighted sum-rate, [7][8].

The single-user PF scheduler has been extensively studied in the literature, see for instance [9][10] where closed-form expressions for the average throughput per user are derived.

This work was supported in part by the European Union through project ROCKET ICT-2007-1-215282 and FEDER funds, and by the Spanish/Catalan Science and Technology Commissions through projects: 2005SGR-00639, TEC2006-06481/TCM, and CONSOLIDER CSD2008-00010 COMONSENS.

A.Agustin, J.Vidal and O.Muñoz are with the Dept. of Signal Theory and Communication. at UPC, Barcelona, Spain. Email: \{adrian.agustin, josep.vidal, olga.munoz\}@upc.edu.
Although in [11] (chapter 6) the benefits of the superposition coding for the multi-user case are pointed out, to the best of our knowledge, the PF scheduler has not been thoroughly studied in that case.

Multi-user schedulers based on weighted sum-rate (WSR) and maximum sum-rate (MSR) criteria have been analyzed in multi-antenna configurations seizing the spatial dimension to serve more users simultaneously. However, when the MSR criterion is applied to the single antenna case, the multi-user scheduler does not offer any gain over the time division multiple access (TDMA) [12], because the best sum-rate is obtained when all resources are allocated to the user with the highest signal-to-noise ratio (SNR).

We look into multi-user schedulers based on the weighted sum-rate (WSR) and proportional fairness (PF) criteria for single-antenna cellular scenario in the downlink. The objective is to group two or three users under unequal channel state and priorities by taking advantage of the large gains that superposition coding offers over the TDMA [11]. We also compare it with multiuser FDMA.

The main contributions of this paper are:

- Analysis of multi-user PF and WSR schedulers under superposed transmissions in the downlink.

- Derivation of criteria for 2-user pairing in both cases.

\section{Signal MOdEL AND SCHEDULERS}

The scenario consists of a base station (BS) and $N_{u}$ mobile stations (MSs), all equipped with single antennas. The BS transmits a pilot signal which is employed by every terminal to measure the current SNR. Afterwards, each receiver feeds back that measurement to the $\mathrm{BS}$. The overhead rate introduced by this feedback is not tackled in this work. The scheduling algorithm considers the channel state of the MSs to select a subset of them (denoted by $U$ ) to be served simultaneously. All the users are waiting for receiving data (full data queues at BS). The three schedulers analyzed are: Qualcomm (or single-user PF), WSR and multi-user PF.

The Qualcomm scheduler selects just one MS on each scheduling instant, maximizing the following metric:

$$
J_{k}^{Q}=\mu_{k} R_{k}
$$

where $\mu_{k}$ describes the weight or priority for user $k$ and $R_{k}$ is the attained bitrate. The selected user is denoted by the subset of size one $U_{Q}$. The achievable bitrate per user is,

$$
R_{k}=\log _{2}\left(1+\rho_{k}\right)
$$

where $\rho_{k}$ stands for SNR at the receiver. The user priority 
introduced in (1) is inversely proportional to the average served throughput, calculated as,

$$
\mu_{i}=\frac{1}{T_{i}}, \quad T_{i}(n)= \begin{cases}(1-\tau) T_{i}(n-1)+\tau R_{i} & \text { if } i \in U_{x} \\ (1-\tau) T_{i}(n-1) & \text { otherwise }\end{cases}
$$

where $T_{i}(n)$ denotes the average throughput at frame $n, R_{i}$ stands for the bitrate of the selected user, $U_{x}$ is equal to $U_{Q}$ and $\tau$ is a tuning parameter that allows varying the performance of the scheduler, from greedy ( $\tau$ close to 0 ) to impose strong user fairness among MSs ( $\tau$ close to 1$)$.

The multi-user PF scheduler selects $K$ users, denoted by the subset $U_{P F}$, maximizing the average served throughput, (3),

$$
J_{U_{P F}}^{P F}=\sum_{j=1}^{\left|U_{P F}\right|} \log \left(T_{j}\right)=\log \left(\prod_{j=1}^{\left|U_{P F}\right|} T_{j}\right)
$$

This criterion has been applied in [13] for multicarrier systems where is shown that can be transformed into,

$$
J_{U_{P F}^{P F}}^{P F}=\log \left(\prod_{j=1}^{\left|U_{P F}\right|}\left(1+\mu_{j} \frac{\tau R_{j}}{1-\tau}\right)\right)
$$

where $\mu_{j}, \tau$ are given in (3) and $R_{j}$ is the bitrate of one selected user, depending on the Gaussian Broadcast channel (BC) [14].

On the other hand, the weighted sum-rate scheduler (WSR) selects $K$ users, referenced by subset $U_{W S R}$, which maximizes,

$$
J_{U_{\text {WSR }}}^{W S R}=\sum_{j=1}^{\left|U_{\text {WSR }}\right|} \mu_{j} R_{j}
$$

where $R_{j}$ denotes the achievable rate of one of the selected users. Those bitrates must lie within the capacity region of the Gaussian broadcast channel when superposed transmissions are envisioned [14] and the power at the BS is distributed among the messages to be transmitted. The user priorities may be calculated according to (3), albeit other criteria can be used to derive them, like limiting the maximum delay or fitting with the maximum stability region [15].

\section{GUIDELINES FOR 2-USER PAIRING}

This section studies the conditions to be satisfied for two users in order to be served under the WSR and PF criteria $\left(\left|U_{W S R}\right|=2,\left|U_{P F}\right|=2\right)$ and yet improve the sum-rate attained under the Qualcomm scheduler. Because of the degradedness of our scenario, we assume there are a strong and a weak user having SNR $\rho_{s}$ and $\rho_{w}\left(\rho_{s} \geq \rho_{w}\right)$ with user priorities $\mu_{s}, \mu_{w}$.

\section{A. Qualcomm $(Q)$}

This scheduler selects the user with the best metric (1),

$$
J_{Q}=\left\{\begin{array}{lc}
\mu_{w} R_{w} & \text { if } \mu_{w} \log _{2}\left(1+\rho_{w}\right) \geq \mu_{s} \log _{2}\left(1+\rho_{s}\right) \\
\mu_{s} R_{s} & \text { otherwise }
\end{array}\right.
$$

where $R_{w}, R_{s}$ are the bitrates, (2), of the weak and strong user. From (7), the condition for selecting only the weak one is,

$$
\rho_{s} \leq\left(1+\rho_{w}\right)^{\frac{\mu_{w}}{\mu_{s}}}-1, \quad \zeta_{Q}=\rho_{s} / \rho_{w}=\left(\left(1+\rho_{w}\right)^{\mu_{w} / \mu_{s}}-1\right) / \rho_{w}
$$

where $\zeta_{Q}$ is the threshold for changing the user selection.

\section{B. Weighted sum-rate (WSR)}

The bitrates must belong to the rate region of the $\mathrm{BC}$ [14],

$$
\left\{\begin{array}{l}
R_{s} \leq \log _{2}\left(1+\gamma \rho_{s}\right) \\
R_{w} \leq \log _{2}\left(1+\rho_{w}\right)-\log _{2}\left(1+\gamma \rho_{w}\right)
\end{array}\right.
$$

where $\gamma$ is the fraction of power allocated to the signal for the strong user, while the other user is allocated $1-\gamma$. Notice that the SNRs $\rho_{s}, \rho_{w}$ are measured from the pilot signal, but they do not correspond to the final SNR of the signals to be decoded whose SNR are $\gamma \rho_{s}$ and $(1-\gamma) \rho_{w}$.

It is known that the maximization of $J^{W S R}$ with $\mu_{s} \geq \mu_{w}$ is obtained when only the strong user is selected. But, for $\mu_{s}<\mu_{w}$ the power allocation depends on the SNR configuration. The maximization of $J^{W S R}$ subject to (9) is derived from,

$$
\frac{\partial J^{W S R}}{\partial \gamma}=\mu_{s} \frac{\rho_{s}}{1+\gamma \rho_{s}}-\mu_{w} \frac{\rho_{w}}{1+\gamma \rho_{w}}=0, \quad \frac{\partial^{2} J^{W S R}}{\partial^{2} \gamma}<0
$$

Thus the optimal power allocation turns out to be,

$$
\begin{aligned}
& \text { if } \mu_{s}>\mu_{w} \quad \gamma=1 \\
& \text { if } \mu_{s} \leq \mu_{w} \quad \gamma=\left\{\begin{array}{lll}
0 & \text { if } & \rho_{s} / \rho_{w} \leq \zeta_{w}^{W S R} \\
\frac{\frac{\rho_{s}}{\rho_{w}}-\frac{\mu_{w}}{\mu_{s}}}{\rho_{s}\left(\mu_{w} / \mu_{s}-1\right)} & \text { if } & \zeta_{w}^{W S R}<\frac{\rho_{s}}{\rho_{w}} \leq \zeta_{s}^{W S R} \\
1 & \text { if } & \rho_{s} / \rho_{w}>\zeta_{s}^{W S R}
\end{array}\right.
\end{aligned}
$$

where $\zeta_{w}^{W S R}, \zeta_{s}^{W S R}$ are the thresholds for scheduling only the weak or only the strong user when $\mu_{s} \leq \mu_{w}$,

$$
\zeta_{w}^{W S R}=\frac{\mu_{w}}{\mu_{s}}, \zeta_{s}^{W S R}=\left\{\begin{array}{cl}
\frac{\mu_{w} / \mu_{s}}{1-\rho_{w}\left(\mu_{w} / \mu_{s}-1\right)} & \text { if } \frac{\mu_{s}}{\mu_{w}}>\frac{\rho_{w}}{1+\rho_{w}} \\
\infty & \text { otherwise }
\end{array}\right.
$$

Applying the optimal power allocation, $J^{W S R}$ results in,

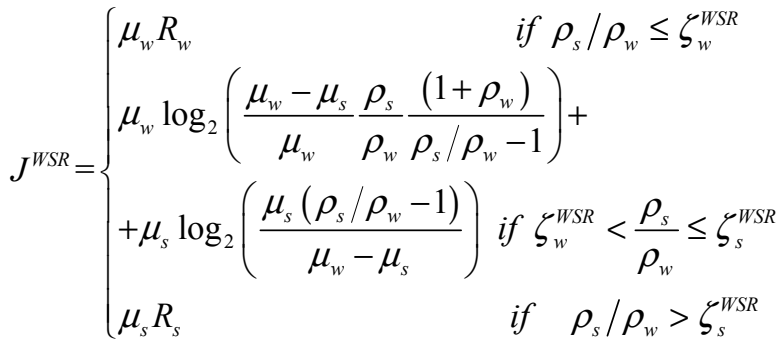

The additive sum-rate gain provided by the WSR over the Qualcomm scheduler is therefore given by,

$$
\Delta^{W S R}= \begin{cases}\log _{2}\left(\frac{\mu_{s}}{\mu_{w}} \frac{\rho_{s}}{\rho_{w}}\right) & \text { if } \zeta_{w}^{W S R}<\frac{\rho_{s}}{\rho_{w}} \leq \zeta_{Q} \\ \log _{2}\left(\frac{\mu_{s}}{\mu_{w}} \frac{\rho_{s}}{\rho_{w}} \frac{1+\rho_{w}}{1+\rho_{s}}\right) & \text { if } \zeta_{Q}<\frac{\rho_{s}}{\rho_{w}} \leq \zeta_{s}^{W S R} \\ 0 & \text { elsewhere }\end{cases}
$$

where $\zeta_{Q}$ subsumes the condition needed from serving the weak over the strong user in the Qualcomm scheduler, (8). Only in the first interval of (14) provides a positive sum-rate gain. Therefore, the 2-user pairing under WSR should satisfy,

$$
\zeta_{w}^{W S R}<\rho_{s} / \rho_{w} \leq \zeta_{Q}
$$




\section{Multiuser Proportional Fairness (PF)}

This scheduler serves two users simultaneously, so that the achievable rates of the users must satisfy the capacity region of the BC. The power at the BS is allocated among the transmitted signals as a result of the maximization of $J^{P F}$ subject to (9). The fraction of power devoted to the strong user ( $\gamma$ ) satisfies the equation,

$$
\begin{aligned}
\frac{\partial J^{P F}}{\partial \gamma}= & \frac{\tau}{1-\tau} \mu_{s} \rho_{s}\left(1+\gamma \rho_{w}\right)\left(1+\mu_{w} \frac{\tau}{1-\tau} \log _{2}\left(\frac{1+\rho_{w}}{1+\gamma \rho_{w}}\right)\right)- \\
& \mu_{w} \frac{\tau}{1-\tau} \rho_{w}\left(1+\gamma \rho_{s}\right)\left(1+\mu_{s} \frac{\tau}{1-\tau} \log _{2}\left(1+\gamma \rho_{s}\right)\right)=0
\end{aligned}
$$

where $\rho_{s}, \rho_{w}$ denote the SNR and $\mu_{s}, \mu_{w}$ are the priorities of the strong and weak user, respectively. The condition to serve only the weak user $(\gamma=0)$ or the strong one $(\gamma=1)$ becomes,

$$
\begin{aligned}
\zeta_{w}^{P F} & =\frac{\rho_{s}}{\rho_{w}}=\frac{\mu_{w} / \mu_{s}}{1+(\tau /(1-\tau)) \mu_{w} \log _{2}\left(1+\rho_{w}\right)}, \\
\zeta_{s}^{P F} & =\mu_{w} / \mu_{s}\left(1+\rho_{s}\right)\left(1+(\tau /(1-\tau)) \mu_{s} \log _{2}\left(1+\rho_{s}\right)\right)-\rho_{s}
\end{aligned}
$$

In terms of sum-rate gain over the Qualcomm scheduler, the gains are positive when the PF is selecting both users while the Qualcomm selects just the weak user. The 2-user pairing criterion for the PF becomes,

$$
\zeta_{w}^{P F}<\rho_{s} / \rho_{w} \leq \zeta_{Q}
$$

Since $\zeta_{w}^{P F}<\zeta_{w}^{W S R}$, the PF criterion offers more opportunities to improve the Qualcomm scheduler than the WSR.

\section{Weighted sum-rate (WSR) under FDMA}

With a slight abuse of the notation, we refer by WSR-FDMA the scheduler that maximizes (6) serving the users orthogonally in frequency. The bitrates of the users are,

$$
\left\{\begin{array}{l}
R_{s} \leq \alpha \log _{2}\left(1+\gamma \rho_{s} / \alpha\right) \\
R_{w} \leq(1-\alpha) \log _{2}\left(1+(1-\gamma) \rho_{w} /(1-\alpha)\right)
\end{array}\right.
$$

where $\alpha$ and $\gamma$ stand for the fraction of bandwidth and power allocated to the strong user, respectively. In this case the variables $\alpha$ and $\gamma$ have to be optimized for maximizing the weighted sum-rate,

$$
\frac{\partial J^{W S R-F D M A}}{\partial \gamma}=0, \quad \gamma=\frac{\alpha}{\rho_{s}} \frac{(1-\alpha)\left(\mu_{s} \rho_{s} / \rho_{w}-\mu_{w}\right)+\mu_{s} \rho_{s}}{\mu_{s} \alpha+\mu_{w}(1-\alpha)}
$$

where $J^{W S R-F D M A}$ considers the weighted sum-rate of (6) using the bitrates defined in (19). By defining,

$$
f(\alpha)=\frac{\alpha+(1-\alpha) \rho_{s} / \rho_{w}+\rho_{s}}{\mu_{s} \alpha+\mu_{w}(1-\alpha)}
$$

and considering (20), the optimal bandwidth allocation becomes

$$
\alpha^{*}=\left\{\begin{array}{l}
\frac{1}{\log (2)} \frac{\mu_{w}\left(1+\rho_{s}\right)-\mu_{w}\left(\rho_{s} / \rho_{w}+\rho_{s}\right)}{\alpha+(1-\alpha) \rho_{s} / \rho_{w}+\rho_{s}}+ \\
+\mu_{s} \log _{2}\left(\frac{\mu_{s} \mu_{w}}{\rho_{s} / \rho_{w}} f^{2}(\alpha)\right)-\log _{2}\left(\frac{\mu_{w} f(\alpha)}{\rho_{s} / \rho_{w}}\right)=0
\end{array}\right.
$$

Since equation (22) has to be solved numerically, we define the following thresholds to identifying which user is served,

$$
\begin{cases}\zeta_{w}^{F D M A}=\rho_{s} / \rho_{w} & \mid \gamma=0, \alpha=0 \\ \zeta_{s}^{F D M A}=\rho_{s} / \rho_{w} & \mid \gamma=1, \alpha=1\end{cases}
$$

The sum-rate gain provided by the WSR-FDMA over the Qualcomm scheduler is given by,

$$
\Delta^{F D M A}=\left\{\begin{array}{c}
\log _{2}\left(\left(\mu_{s}\right)^{\alpha}\left(\frac{\mu_{w}}{\rho_{s} / \rho_{w}}\right)^{(1-\alpha)} \frac{f(\alpha)}{1+\rho_{w}}\right) \text { if } \zeta_{w}^{F D M A} \leq \frac{\rho_{s}}{\rho_{w}} \leq \zeta_{Q} \\
\log _{2}\left(\left(\mu_{s}\right)^{\alpha}\left(\frac{\mu_{w}}{\rho_{s} / \rho_{w}}\right)^{(1-\alpha)} \frac{f(\alpha)}{1+\rho_{s}}\right) \text { if } \zeta_{Q}<\frac{\rho_{s}}{\rho_{w}} \leq \zeta_{s}^{F D M A} \\
0 \quad \text { elsewhere }
\end{array}\right.
$$

where $\zeta_{Q}$ is defined in (8) and function $f$ in (21).

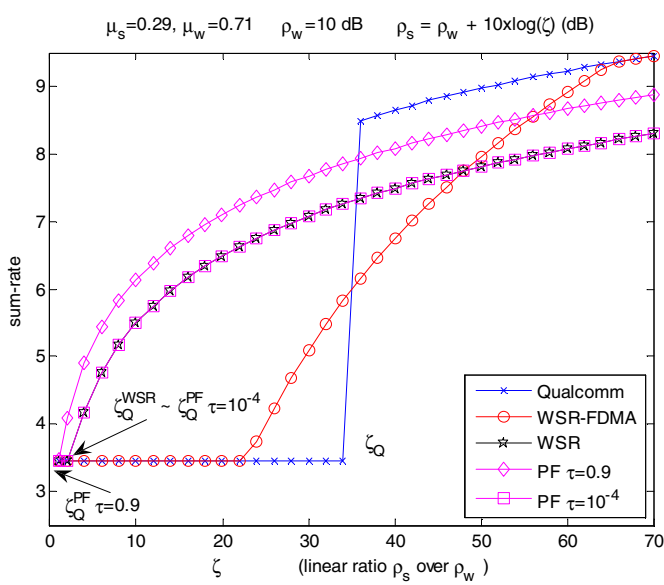

Fig. 1. Sum-rate provided by the Qualcomm, WSR, WSR-FDMA and PF with $\tau=\left\{0.9,10^{-4}\right\}$ as a function of the linear ratio $\zeta=\rho_{s} / \rho_{w}$. User priorities $\mu_{s}=0.29, \mu_{w}=0.71, \rho_{w}=10 \mathrm{~dB}, \rho_{s}=\rho_{w}+10 \times \log _{10}(\zeta)$.

In order to compare the performance of the different schedulers, Fig. 1 presents the sum-rate attained by each one for a configuration where the user-priorities are fixed to $\mu_{s}=0.29, \mu_{w}=0.71$, the weak user has a SNR equal to $\rho_{w}=10 \mathrm{~dB}$ and the SNR of the strong user varies with the parameter $\zeta$ as $\rho_{s}=\rho_{w}+10 \times \log _{10}(\zeta)$. We can observe that the Qualcomm scheduler selects either the weak or the strong user at $\zeta_{Q}$. In the latter case, the sum-rate increases with $\zeta$ because the SNR of the strong user also increases. For the WSR-FDMA three different performances are observed: only the weak user $(\zeta \leq 22)$, both, weak and strong (orthogonally), or just the strong user $(\zeta \geq 66)$ are served. For the WSR and PF the conditions for serving just the strong user are not satisfied. The maximum gain with respect to the Qualcomm scheduler is attained at $\zeta=\zeta_{Q}$, i.e. while the Qualcomm scheduler selects the weak user, the other schedulers are selecting both users. Finally, for the PF scheduler, we sketch the performance for two values of $\tau$. When $\tau$ is close to zero the WSR and PF get the same performance, but for $\tau$ values close to one, there are significant differences in terms of sum-rate.

Fig. 1 showed that Qualcomm scheduler is superior under some circumstances. That seems to contradict the theory, which states that achievable rate region of TDMA (Qualcomm) is in general lower than FDMA (WSR-FDMA) and $\mathrm{BC}$ (WSR, PF). However, the sum-rate provided by each scheduler depends on the user priority, $\mu_{s}, \mu_{w}$. Fig. 2 illustrates 
a configuration where the TDMA gets the best sum-rate. Fig. 2 assumes the same configuration as in Fig. 1 with $\zeta=40$.

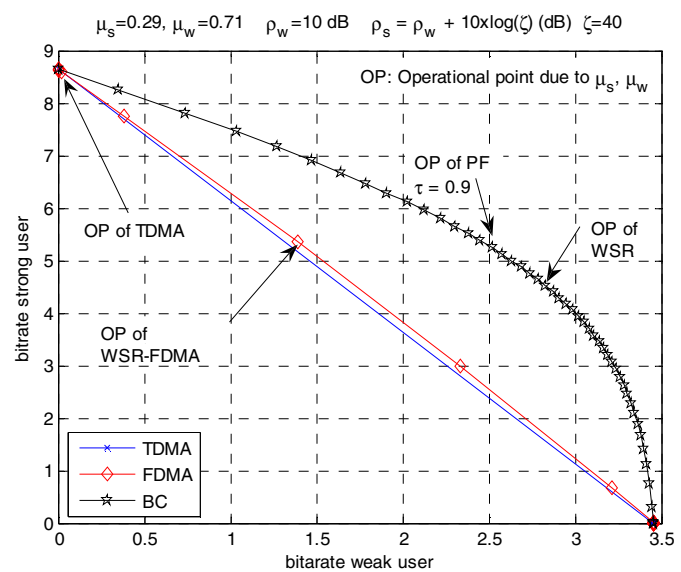

Fig. 2. Achievable rate region of TDMA (Qualcomm), FDMA (WSRFDMA) and BC (WSR and PF). User priorities $\mu_{s}=0.29, \mu_{w}=0.71, \rho_{w}=10 \mathrm{~dB}$, $\rho_{s}=26.02 \mathrm{~dB}(\zeta=40)$.

\section{Cellular Evaluation}

The evaluation of the Qualcomm, WSR and PF schedulers has been done in a simplified scenario where the MSs are uniformly distributed in a cell of radius $r=200$ meters, being the BS at the cell centre. The pathloss exponent is 3 . The noise power, transmitted power and other constants have been configured so as to get $\mathrm{SNR}=0 \mathrm{~dB}$ when a MS is at the cell edge and $30 \mathrm{~dB}$ when the user is close to the BS. The channel coefficients are Rayleigh distributed, constant over a frame and independent among users. The simulation evaluates 300 user deployments, each one consisting of $N_{u}$ users and simulated during 8000 frames. Users feed back their current SNR to the BS. The selected users maximize the different scheduling criteria, where their priorities are given by (3).

Since we are dealing with multiple users under superposition coding strategy, the scheduler must evaluate all the combinations for the user pairing. This task is tractable for the 2-user WSR because there is a closed-form expression for the metric to be maximized, (13), albeit for more users or the multi-user PF scheduler the task becomes cumbersome. To this end, and following similar steps as in [7], we consider a candidate list $\Gamma$ with $L$ users with the best metric $\mu_{k} R_{k}$,

$$
\Gamma=\left\{k \in\left[1, N_{u}\right] \quad \mid \quad \mu_{k} R_{k} \geq \eta\right\}, \quad|\Gamma|=L
$$

where $\mu_{k}$ and $R_{k}$ are the priority and the bitrate of the $k$-th user assuming that the BS allocates all the power to that user, (2).

The schedulers are compared in terms of user throughput, cell throughput and delay, defining delay as the average number of frames elapsed between two consecutive selection instants of the same user.

\section{A. Per-user throughput and delay for 2-user schedulers}

This subsection studies the benefits in terms of throughput and delay introduced by the 2-user WSR and PF schedulers in a scenario with $N_{u}=30 \mathrm{MSs}$. Fig. 3 depicts the $10 \%$-outage throughput per user as a function of $\tau$ for the Qualcomm, 2user WSR and PF with $L=\{5,9\}$. Notice that in order to obtain that measure we have considered all users present in the cell. When $\tau$ goes to zero the $10 \%$-outage throughput per user tends to zero. This performance is caused by the selection of the users with the best channel conditions. In such a case, the remaining users are rarely served, imposing a very low throughput per user. Accordingly, when $\tau$ increases more users are served and the throughput improves. But at a certain point ( $\tau=5 \times 10^{-3}$ in Fig. 3 ) the $10 \%$-outage throughput per user decreases as $\tau$ goes to 1 . This is a consequence of serving the selection of deprived users in the cell due to their high user priority, which becomes more important than the current channel of the user. The best channel users, which could get larger bitrates, are less frequently selected. It is important to remark that the 2-user schedulers (WSR and PF) outperform the Qualcomm scheduler, except for the 2-user WSR with $\tau \gg 0.1$. In that latter case, we have observed (but not showed here) that the average number of served users decreases, explaining the peculiar behavior of 2-user WSR depicted Fig. 3. Finally, the performance of the 2-user PF is almost independently of the value of $\Gamma$ for $L=\{5,9\}$.

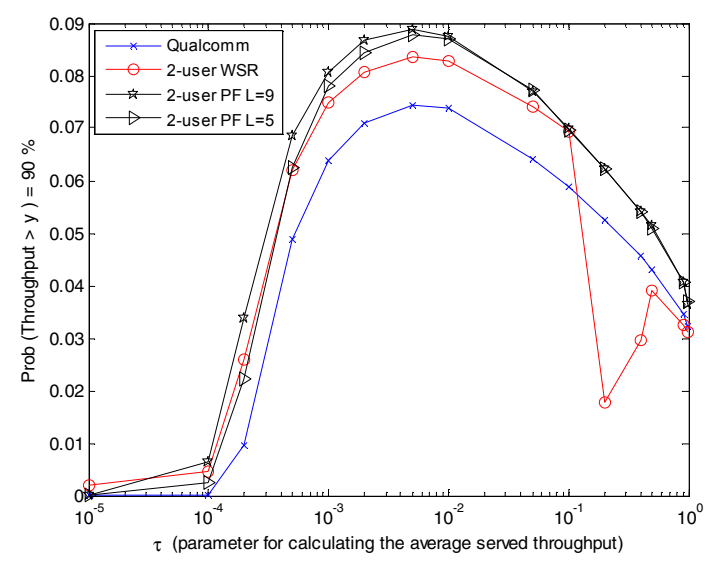

Fig. 3. 10\%-outage throughput per user as a function of $\tau$ for the Qualcomm, 2-user WSR and PF ( with $L=\{5,9\}$ ) schedulers. $N_{u}=30$ MSs.

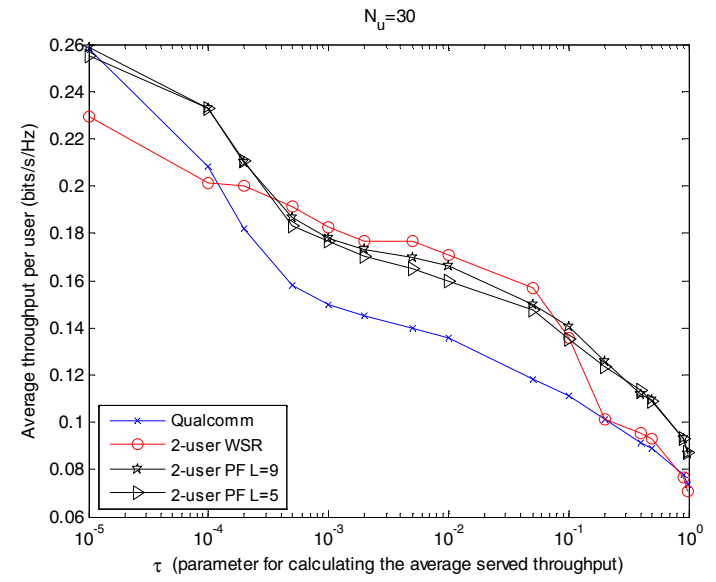

Fig. 4. Average throughput per user as a function of $\tau$, for the Qualcomm, 2user WSR and PF (with $L=\{5,9\}$ ) schedulers. $N_{u}=30$ MSs.

The average throughput per user is sketched in Fig. 4 as a function of $\tau$. The average throughput decreases monotonically as $\tau$ goes to 1 in all cases. Notice that for low values of $\tau$ there is a significant difference between the average and the 10\%-outage throughput (Fig. 3). That means 
that user throughput presents a disperse statistic as a consequence of having a small number of users in good SNR conditions. Likewise, the 2-user PF scheduler always improves the Qualcomm scheduler while the 2-user WSR is clearly inferior to 2-user PF for values of $\tau$ close to zero (greedy) and close to one (round robin).

The 90\%-outage per-user delay (in log scale) is presented in Fig. 5. For low values of $\tau$, large delays are found because only a small number of users are served. As $\tau$ increases the Qualcomm scheduler tends to get a constant delay of 30 frames. The 2-user PF reduces to one half the delay while the WSR scheduler achieves some intermediate delay values.

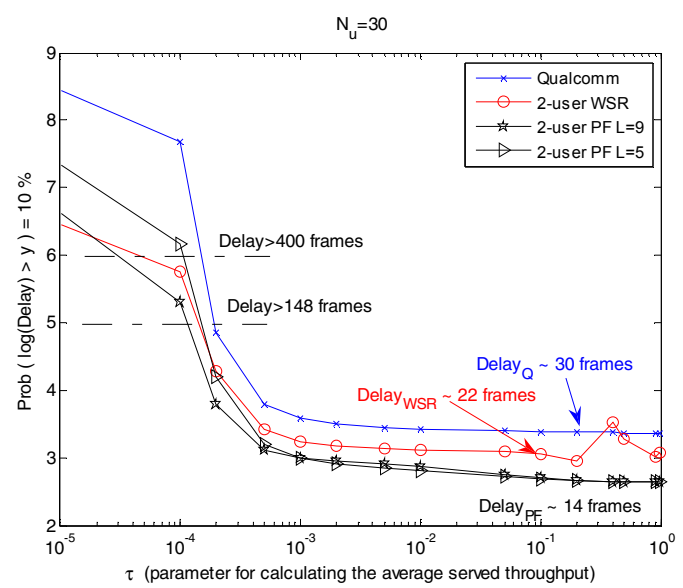

Fig. 5. 90\%-outage delay (logarithm scale) per user as a function of $\tau$ for the Qualcomm, 2-user WSR and PF (with $L=\{5,9\}$ ) schedulers. $N_{u}=30 \mathrm{MSs}$

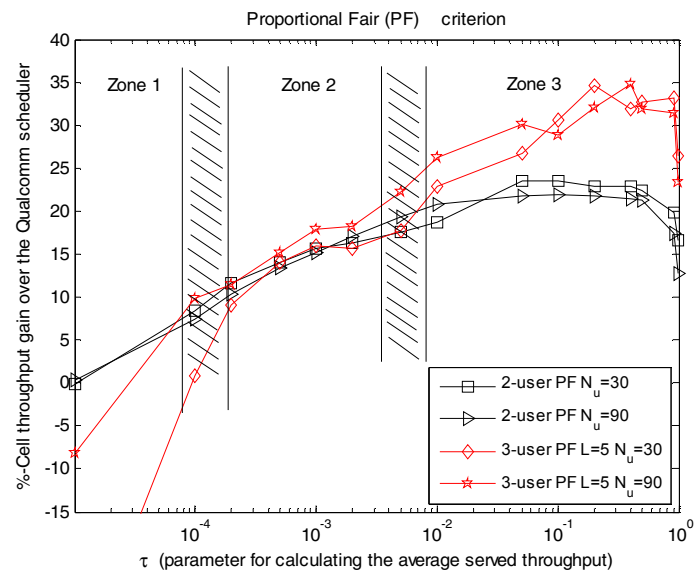

Fig. 6. Percentage-wise cell throughput gain over the Qualcomm scheduler as a function of $\tau$ for the $\{2,3\}$-user PF with $L=5$ schedulers. $N_{u}=\{30,90\}$ MSs.

\section{B. Cell throughput with 2-user and 3-user PF schedulers}

Here we investigate cell throughput gain obtained by multiuser PF schedulers (2-user and 3-user) over the Qualcomm scheduler. The 3-user PF scheduler designs the bit rates and power allocation by maximizing (5) taking into account the achievable rate regions of the 3-user $\mathrm{BC}$ [14]. A candidate list of users with $L=5$ is assumed. Fig. 6 presents the percentagewise cell throughput gain for the PF scheduler with $N_{u}=\{30,90\}$ which can be divided in three zones. The 2-user scheduler gets the best throughput in zone 1, while 3-user scheduler in zone 3 (a gain around 34\%). In zone 2, both schedulers attain a similar performance. Although not shown, it has been observed than the delay for the 3-user scheduler is $1 / 3$ the delay of the Qualcomm scheduler in zones 2 and 3.

\section{CONCLUSIONS}

This work analyzes multi-user schedulers in the Gaussian BC based on superposition coding. The WSR and PF scheduling criteria are compared with the Qualcomm scheduler (single-user PF) in terms of throughput and delay. User pairing criteria based on SNR and user priority are presented. Results have shown that by serving multiple-users under the PF criterion, the delay per user is improved, but the throughput depends on the value of $\tau$, which tunes the performance of the scheduler. In the setup described for greedy schedulers ( $\tau$ close to 0 ) it is preferable to schedule 2users, while if fairer criterion is adopted ( $\tau$ close to 1 ), 3-user scheduling is better. The performance is in all cases improved with respect to the Qualcomm scheduler. The WSR scheduler is able to get a similar performance than PF scheduler just for certain intermediate values of $\tau$.

\section{REFERENCES}

[1] R.Knopp, P.Humblet, "Information capacity and power control in single cell multiuser communications", in Proc. IEEE Intl. Conf. on Communications (ICC), 1995.

[2] F.P.Kelly, A.K:Maulloo, D.K.H.Tan, "Rate control in communication networks: shadow prices, proportional fairness and stability", Journal of the Operational Research Society, vol.49, April 1998.

[3] A.Jalali, R.Padovani, R.Pankai, "Data throughput of CDMA HDR a high efficiency-high data rate personal communication wireless system", in Proc. IEEE Vehicular Techn. Conf. (VTC) Spring, 2000.

[4] P.Viswanath, D.N.C.Tse, R.Laroia, "Opportunistic beamforming using dumb antennas", IEEE Trans. on Information Theory, vol.48, no.6, June 2002.

[5] J.Mo, J.Walrand, "Fair End-to-end window-based congestion control", IEEE/ACM Trans. Networking, vol.8, no.5, Oct. 2000.

[6] J.Liu, Y.T.Hou, "Weighted Proportional Fairness Capacity of Gaussian MIMO Broadcast channels", in Proc. IEEE Conf. on Computer Comm. (INFOCOM), 2008.

[7] H.Viswanathan, S.Venkatesan, H.Huang, "Downlink Capacity evaluation of cellular networks with known-interference cancellation", IEEE Journal on Selec. Areas in Comm., vol.21, no.5, June 2003.

[8] K.Jagannathan, S.Borst, P.Whiting, E.Modiano, "Scheduling of Multiantenna Broadcast systems with heterogeneous users", IEEE Journal on Selec. Areas in Comm., vol.25, no.7, Sept. 2007.

[9] J.G.Choi, S.Bahk, "Cell-Throughput analysis of the proportional fair scheduler in the single-cell environment", IEEE Trans. on Vehicular Techn., vol.56, no.2, March 2007.

[10] E.Liu, K.K.Leung, "Fair Resource allocation under Rayleigh and/or Rician fading environments", in Proc. IEEE Personal Indoor Mobile Radio Communications (PIMRC), Sept. 2008.

[11] D.Tse, P.Viswanath, Fundamentals of Wireless Communication, Cambridge University Press, 2005.

[12] N.Jindal, A.Goldsmith, "Dirty-Paper coding versus TMDA for MIMO Broadcast Channels", IEEE Trans. on Information Theory, vol.51, no.5, May 2005.

[13] H.Kim, Y.Han, "A Proportional Fair scheduling for multi-carrier transmission systems", IEEE Comm. Letters, vol.9, no.3, March 2005.

[14] T.M.Cover, J.A.Thomas, Elements of Information Theory, 2nd edition, Wiley, 1991.

[15] G.Song, "Joint channel-aware and queue-aware data scheduling in multiple shared wireless channels", Proc. of IEEE Wireless Conf. of Networking and Comm. (WCNC), March 2004. 